\title{
Estilo de interacción del entrenador, clima motivacional percibido y satisfacción de las necesidades psicológicas básicas en futbolistas jóvenes
}

\author{
Coach interaction style, perceived motivational climate and satisfaction of \\ basic psychological needs in young soccer players
}

\section{Estilo de interação do treinador, clima motivacional percebido e satisfação das necessidades psicológicas básicas em jovens futebolistas}

\author{
Moreno-Luque, M., Reigal, R. E., Morillo-Baro, J. P., Morales-Sánchez, V., Hernández-Mendo, A. \\ Universidad de Málaga
}

\begin{abstract}
RESUMEN
El objetivo del presente trabajo fue analizar el clima motivacional percibido a través de las relaciones existentes entre el estilo de interacción del entrenador (apoyo a la autonomía o estilo controlador) con los constructos de la Teoría de las Metas de Logro (clima ego o clima tarea) y el grado de satisfacción con las necesidades psicológicas básicas en una muestra de jóvenes deportistas. Participaron 125 futbolistas de la provincia de Málaga (España), con edades comprendidas entre los 15 y 18 años $(M=16.66, D T=1.13)$. Para recoger la información se utilizó la Escala de Estilo Controlador del Entrenador (CCBS), el Cuestionario de Percepción de Soporte de la Autonomía en el Proceso de Entrenamiento (ASCQ), el Cuestionario de Clima Motivacional Percibido en el Deporte (PMCSQ-2) y Escala de Medición de la Satisfacción de las Necesidades Psicológicas en el Ejercicio (PNSE). Los análisis efectuados pusieron de manifiesto relaciones negativas entre la satisfacción de las necesidades psicológicas básicas y el estilo controlador, pero positivas con el apoyo a la autonomía. Además, destaca las relaciones positivas entre el estilo controlador y la percepción del clima motivacional de implicación en el ego, siendo negativas con el clima de implicación en la tarea.
\end{abstract}

Palabras clave: clima motivacional; bienestar; autonomía; estilo controlador; fútbol.

\begin{abstract}
The aim of this paper was to analyze the perceived motivational climate through the relationships between the coach's interaction style (autonomy support or controlling style) with the constructs of the Achievement Goal Theory (ego climate or task climate) and the degree of basic psychological needs satisfaction in a sample of young athletes. 125 players from the province of Málaga (Spain) participated in this research, aged between 15 and 18 years old $(M=16.66, S D=1.13)$. To collect the information we used the Controlling Coach Behaviors Scale (CCBS), the Autonomy-Supportive Coaching Questionnaire (ASCQ), the Perceived Motivational Climate in Sport Questionnaire - 2 (PMCSQ-2) and the Psychological Need Satisfaction in Exercise scale (PNSE). Results revealed negative relationships between the satisfaction of basic psychological needs and the controlling style, but positive
\end{abstract}




\section{Moreno-Luque, M., Reigal, R. E., Morillo-Baro, J. P., Morales-Sánchez, V., Hernández-Mendo, A.}

with autonomy support. In addition, it highlights the positive relationships between the controlling style and the perception of ego-involving climates, being negative with the task-involving climates.

Keywords: motivational climate; welfare; autonomy; controlling style; soccer.

\section{RESUMO}

O objetivo deste estudo foi analisar o clima motivacional percebido através das relações entre treinador estilo de interação (apoio para a autonomia ou estilo controlador) com as construções da Teoria da Goal Achievement (clima ego ou o clima tarefa e o grau de satisfação com necessidades psicológicas básicas em uma amostra de jovens atletas. Participaram 125 jogadores da província de Málaga (Espanha), com idades compreendidas entre os 15 e os 18 anos $(\mathrm{M}=16,66, \mathrm{DT}=1,13)$. Escala Estilo driver Trainer $(\mathrm{CCBS})$ foi usado para coletar informações, Perceptions Questionnaire apoiar a autonomia no processo de formação (ASCQ) Questionário Percebida Motivacional Climate in Sport (PMCSQ-2) e Escala de Medida da Satisfação das Necessidades Psicológicas no Exercício (PNSE). As análises realizadas revelaram relações negativas entre a satisfação das necessidades psicológicas básicas e o estilo controlador, mas positivas com o apoio à autonomia. Além disso, destaca as relações positivas entre o estilo controlador e a percepção do clima motivacional de envolvimento no ego, sendo negativo com o clima de envolvimento na tarefa.

Palavras chave: clima motivacional; bem-estar; autonomía; estilo controlador; futebol.

\section{INTRODUCCIÓN}

El deporte representa una de las actividades de ocio que más beneficios tiene para el desarrollo físico y psicosocial de las personas (Bullock, y Giesbrecht, 2014; Rhodes, Janssen, Bredin, Warburton, y Bauman, 2017; Warburton, y Bredin, 2017). Por ello, favorecer la adherencia a este tipo de prácticas es interesante para favorecer el desarrollo integral de niños y adolescentes. Bajo estas circunstancias, resulta de gran interés conocer cuáles son los aspectos que pueden determinar una mayor motivación en los jóvenes deportistas y cómo este tipo de tareas pueden ser más satisfactorias para ellos. En este sentido, una de las piezas claves para generar un adecuado clima motivacional en los deportistas es el comportamiento del los entrenadores y qué tipo de interacción establecen con sus jugadores (BarbosaLuna, Tristán, Tomás, González, y López-Walle, 2017; Torregrosa, Viladrich, Ramis, Azócar, Latinjak, y Cruz, 2010).

Para analizar el impacto que tiene la conducta del entrenador en la motivación de los deportistas, la Teoría de la Autodeterminación (TDA) y la Teoría de las Metas de Logro (TML) son dos de los contextos teóricos que más repercusión tienen en la actualidad, siendo el marco fundamental en el que se han desarrollado numerosas investigaciones en el deporte de formación (e.g., Castillo, Duda, Álvarez, Mercé y Balaguer, 2011). La TAD (Deci y Ryan, 1985, 2000) se centra básicamente en evaluar el nivel en que la conducta de las personas es autodeterminada, y se explica a partir de seis mini-teorías: (1) Teoría de la Evaluación Cognitiva (Cognitive Evaluation Theory); (2) Teoría de la Integración Organísmica (Organismic Integration Theory); (3) Teoría de las Orientaciones de Causalidad (Causality Orientations Theory); (4) Teoría de las Necesidades Psicológicas Básicas (Basic Psychological Needs Theory); (5) Teoría de los Contenidos de Meta (Goal Contents Theory); (6) Teoría de Motivación de las Relaciones (Relationships Motivation Theory).

Específicamente, la teoría de las Necesidades Psicológicas Básicas (NPB) es una de la que posee mayor apoyo empírico, siendo analizada en múltiples trabajos (Balaguer et al., 2012; González, Tomás, Castillo, Duda, y Balaguer, 2017; Orkibi, y Ronen, 2017; Sylvester, Curran, Standage, Sabiston, y Beauchamp, 2018). Dicha teoría considera que el ser humano debe satisfacer las necesidades de competencia, autonomía y relaciones sociales, lo cual se ha relacionado positivamente con la salud psicológica y el bienestar (Moreno-Murcia, Marzo, Martínez-Galindo, y Conte, 2011).

Por otro lado, la TML (Nicholls, 1989; Duda y Nicholls, 1992), considera que la conducta de las personas es propositiva, organizando sus esfuerzos para alcanzar una meta determinada. Desde este enfoque, la actuación del deportista podría estar dirigida hacia el ego o la tarea, las cuales no son excluyentes y pueden coexistir. Cuando se orienta al 


\section{Clima motivacional y necesidades psicológicas básicas en futbolistas jóvenes}

ego, primaría la obtención de buenos resultados deportivos, el criterio de éxito se situaría en la comparación con otros deportistas y se predominaría el reconocimiento externo. Cuando se orienta a la tarea, el criterio de mejora es interno, primando el esfuerzo por la mejora personal y la satisfacción con la propia práctica deportiva, más allá de focalizar la actividad en el resultado obtenido (Nicholls, 1989). Se considera que la orientación a la tarea incrementa los niveles de motivación intrínseca, fortalece el compromiso con la actividad que se está realizando, protege ante obstáculos que puedan encontrarse y favorecería los procesos de aprendizaje (Duda 2001; Fabra, Balaguer, Castillo, Mercé y Duda, 2013).

La figura del entrenador ha sido uno de los agentes sociales más estudiados en el contexto deportivo (Adame-Garza, Tristán-Rodríguez, Tomás, Hernández-Mendo, y López-Walle, 2017; Smith, Quested, Appleton, y Duda, 2017). Desde la TML se considera que son figuras esenciales en la creación de climas motivacionales que favorezcan la orientación a la tarea o al ego (Duda y Balaguer, 2007). Cuando el entrenador valora el esfuerzo de sus deportistas, focaliza su atención en aspectos del aprendizaje, tiene en cuenta su punto de vista y promueve relaciones personales positivas, incrementa la probabilidad de que se desarrolle un clima de implicación en la tarea. Cuando el entrenador pone énfasis en el resultado, en los errores de sus jugadores en vez de en el progreso o no se preocupa por las relaciones personales entre sus deportistas, aumentaría la posibilidad de que se genere un clima de implicación en el ego (Newton, Duda y Yin, 2000; Olympiou, Jowett, y Duda, 2008). $\mathrm{Se}$ considera que un clima motivacional de implicación en la tarea promueve un mayor nivel de autoestima, satisfacción, bienestar, adherencia y disfrute con la práctica deportiva entre los deportistas (Atkins, Johnson, Force, y Petrie, 2015; Castillo et al., 2011; Jaakkola, Ntoumanis, y Liukkonen, 2016).

En la manera que tiene el entrenador de interactuar con sus jugadores, se pueden diferenciar conductas que conforman un estilo más controlador frente a otro que apoye más a la autonomía. Aquellos que son controladores pueden perjudicar a sus deportistas impidiendo satisfacer sus NPB, disminuyendo su bienestar o provocando en ellos una experiencia menos positiva (Castillo, González, Fabra, Mercé, y Balaguer, 2012; Mars, Castillo, López-Walle, y Balaguer, 2017). Para favorecer la autonomía en estos contextos, el entrenador debe ser flexible, aceptar las opiniones de otros, ofrecer información adecuada al contexto y a la tarea que están desarrollando o mostrar empatía con el deportista (Deci y Ryan, 1987; Bartholomew, Ntoumanis y Thøgersen-Ntoumani, 2010). Cuando se utilizan este tipo de estilo interpersonal, se incrementa la posibilidad de mejorar el funcionamiento del deportista y favorecer el disfrute con la práctica realizada (Schüler, Wegner y Knechtle, 2014).

En cualquier contexto, las personas tienden a implicarse en las tareas que realizan si consideran que tienen cierto control sobre el ambiente y que permite desarrollar sus capacidades. Por el contrario, cuando se ejerce demasiada presión sobre la tarea y existe un excesivo control sobre la conducta, la tendencia a implicarse será menor y las posibilidades de abandonar la tarea, disfrutar con ellas y obtener cierto grado de bienestar disminuirá (e.g., Balaguer et al., 2012). Por ello, se considera que un estilo interpersonal que promueva la autonomía será más favorable para que se satisfagan las NPB (Álvarez, Castillo, Duda y Balaguer, 2009; Balaguer et al., 2012; García, Sánchez-Miguel, Leo-Marcos, Sánchez-Oliva y Amado, 2012) y se incremente el bienestar, pero también para que la percepción del clima motivacional sea de mayor implicación en la tarea que al ego, disfrutando más del propio aprendizaje deportivo.

El propósito de esta investigación fue analizar las relaciones que existen entre el estilo interpersonal de apoyo a la autonomía o el estilo controlador, con la percepción del clima motivacional generado por los entrenadores/as (ego vs tarea) y el grado de satisfacción de las NPB en un grupo de futbolistas jóvenes.

\section{MATERIAL Y MÉTODOS}

\section{Participantes}

En el estudio participaron 125 jóvenes con edades comprendidas entre los 15 y 18 años. $(M=16.66$; $D T=1.13)$. El $58.40 \%$ eran de género masculino $(n=$ $73)$ y el $41.60 \%$ eran de género femenino $(n=52)$. Todos ellos/as jugadores de fútbol no profesional de las categorías cadetes y juveniles, pertenecientes a diversos clubes de la provincia de Málaga. Como criterio de inclusión y exclusión, además de pertenecer a la categoría cadete o juvenil, debían llevar al menos una temporada en el equipo y no 
Moreno-Luque, M., Reigal, R. E., Morillo-Baro, J. P., Morales-Sánchez, V., Hernández-Mendo, A.

haber cambiado de entrenador en el último año, ya que para el estudio era primordial que conociesen bien a sus entrenadores.

\section{Instrumentos}

a) Cuestionario de Clima Motivacional Percibido
en
el

(Balaguer, Guivernau, Duda y Crespo, 1997). Este cuestionario, constituido por 24 ítems, valora el grado en que los deportistas perciben si el clima motivacional creado por el entrenador es de implicación a la tarea (e.g., El entrenador le da importancia al esfuerzo personal de los jugadores) o de implicación al ego (e.g., El entrenador deja claro quiénes son los mejores). Se responde con una escala Likert de cinco opciones, desde 1 "muy en desacuerdo" hasta 5 "muy de acuerdo". Los análisis de consistencia interna de la escala ofrecieron para este trabajo un Alfa de Cronbach con valores similares para cada uno de los factores: (Implicación en el ego $=.87$ e Implicación en la tarea $=.84$ ).

b) Escala de Medición de la Satisfacción de las Necesidades Psicológicas en el Ejercicio (PNSE) de Wilson, Rogers, Rodgers, y Wild (2006), en su versión validada al español por Moreno-Murcia et al. (2011). La PNSE consta de 18 ítems y tres factores: competencia (e.g., En mis entrenamientos yo creo que puedo completar los ejercicios que son un reto personal), autonomía (e.g., En mis entrenamientos siento que puedo hacer ejercicios a mi manera) y relación con los demás (e.g., En mis entrenamientos me siento atado a mis compañeros de ejercicios porque ellos me aceptan por quien soy). Se responde con una escala Likert de seis opciones, desde 1 "falso" hasta 6 "verdadero". Los análisis de consistencia interna de la escala ofrecieron para este trabajo un Alfa de Cronbach con valores similares para cada uno de los factores: (Competencia $=.89$; Autonomía $=.77$ y Relación con los demás= .77).

c) Escala de Estilo Controlador del Entrenador (CCBS), de Bartholomew et al. (2010), validada al contexto español por Castillo et al. (2014). Esta escala analiza la manera que tiene el entrenador de controlar a los deportistas durante los entrenamientos. Está formado por 15 ítems y cuatro factores: atención condicional negativa (e.g., Mi entrenador amenaza con castigarme para "mantenerme a raya" durante el entrenamiento), el uso controlador de recompensas (e.g., Mi entrenador solo utiliza premios y/o halagos para hacerme entrenar más duro), el uso de la intimidación (e.g., Mi entrenador me grita delante de los otros para que haga determinadas cosas) y el excesivo control personal (e.g., Mi entrenador trata de entrometerse en aspectos de mi vida fuera del fútbol). Se responde con una escala Likert de siete opciones, desde 1 "totalmente en desacuerdo" hasta 7 "totalmente de acuerdo". Los análisis de consistencia interna de la escala ofrecieron para este trabajo un Alfa de Cronbach con valores similares para cada uno de los factores: (Uso controlador de recompensas $=.75$; Atención condicional negativa $=$ .71; Intimidación $=.85$ y Excesivo control personal $=$ $.82)$.

d) Cuestionario de Percepción de Soporte de la Autonomía en el Proceso de Entrenamiento (ASCQ) de Conroy y Coatsworth (2006), en su versión validada al español (Conde et al., 2010). Compuesto por 9 ítems, está dividido en dos dimensiones: interés en la opinión del deportista (e.g., Mi entrenador me pide mi opinión sobre lo que quiero hacer en el entrenamiento) y valoración del comportamiento autónomo (e.g., Mi entrenador me valora por las decisiones que tomo en el entrenamiento). Se responde con una escala Likert de siete opciones, desde 1 "totalmente en desacuerdo" hasta 7 "totalmente de acuerdo". Los análisis de consistencia interna de la escala ofrecieron para este trabajo un Alfa de Cronbach con valores similares para cada uno de los factores: (Interés en la opinión del deportista $=.86$ y Valoración del comportamiento autónomo $=.72$ ).

\section{Procedimiento}

La captación de la muestra se realizó mediante reuniones con los responsables de los equipos participantes; en ellas se les pidió colaboración, se les explicó la finalidad del estudio, se obtuvo el consentimiento informado de todos y el permiso de los padres de los participantes menores de edad. Los datos fueron recogidos antes de los entrenamientos. El tiempo estimado para la cumplimentación de los cuestionarios fue de 45 minutos aproximadamente. Durante su cumplimentación se estuvo presente para solucionar cualquier duda que pudiera plantearse. Al terminar, se agradeció su participación y se les indicó que podrían recibir un informe con los resultados obtenidos en la evaluación. Además, durante todo el 


\section{Clima motivacional y necesidades psicológicas básicas en futbolistas jóvenes}

proceso de investigación se respetaron los principios éticos de la declaración de Helsinki (World Medical Association, 2013).

\section{Análisis estadístico}

Los datos fueron sometidos a análisis descriptivos e inferenciales. Se comprobó la normalidad de los mismos (Kolmogorov-Smirnov) y la consistencia interna de las escalas (Alfa de Cronbach). Para analizar las correlaciones entre variables se utilizó el coeficiente bivariado de Pearson. La capacidad predictiva del estilo controlador o de apoyo a la autonomía sobre la satisfacción de las NPB y el clima motivacional se evaluó mediante análisis de regresión lineal (pasos sucesivos). Para el procesamiento estadístico de los datos se utilizó el programa informatizado SPSS en su versión 23.0.

\section{RESULTADOS}

La tabla 1 muestra los estadísticos descriptivos y de normalidad de las variables objeto de estudio.

Tabla 1

Estadísticos descriptivos y análisis de normalidad.

\begin{tabular}{lccccc}
\hline & Media & DT & Asimetría & Curtosis & $\boldsymbol{Z}$ \\
\hline Necesidad competencia & 4.85 & .95 & -.90 & .38 & 1.35 \\
Necesidad autonomía & 4.31 & 1.08 & -.79 & .54 & 1.18 \\
Necesidad relación con los demás & 4.56 & .89 & -.36 & -.13 & .92 \\
Clima ego & 2.74 & .86 & -.02 & -.38 & .61 \\
Clima tarea & 3.99 & .61 & -.57 & -.12 & .98 \\
Uso recompensas & 3.13 & 1.34 & -.09 & -.88 & 1.24 \\
Atención condición negativa & 2.89 & 1.23 & -.11 & -1.08 & 1.13 \\
Intimidación & 2.59 & 1.41 & .50 & -.80 & 1.27 \\
Control personal & 2.63 & 1.52 & .77 & -.35 & .98 \\
Interés opinión deportista & 4.17 & 1.39 & -.17 & -.37 & .80 \\
Valoración comportamiento autónomo & 4.78 & 1.19 & -.25 & -.04 & 1.03 \\
\hline
\end{tabular}

NOTA: $Z=$ Kolmogorov-Smirnov.

En la tabla 2 se presentan los coeficientes de correlación bivariado de Pearson. Como se puede observar, han existido relaciones significativas y de carácter negativo entre el estilo controlador del entrenador con la satisfacción de la necesidad de relación con los demás y el clima motivacional de implicación en la tarea, y positiva con el clima motivación de implicación en el ego. Asimismo, el estilo controlador, menos su dimensión atención hacia la condición negativa, se ha relacionado negativamente con la satisfacción de la competencia. Por otro lado, el interés sobre la opinión del deportista ha correlacionado positivamente con la satisfacción de la autonomía, y la valoración del comportamiento autónomo con la satisfacción de las tres necesidades psicológicas básicas. El nivel de correlación más elevado se ha producido entre el estilo controlador del entrenador y el clima motivacional con implicación hacia el ego $(p<.001)$. 
Moreno-Luque, M., Reigal, R. E., Morillo-Baro, J. P., Morales-Sánchez, V., Hernández-Mendo, A.

Tabla 2

Análisis de correlación (Pearson)

\begin{tabular}{lccccc}
\hline & $\begin{array}{c}\text { Necesidad } \\
\text { competencia }\end{array}$ & $\begin{array}{c}\text { Necesidad } \\
\text { autonomía }\end{array}$ & $\begin{array}{c}\text { Necesidad } \\
\text { relación con } \\
\text { los demás }\end{array}$ & $\begin{array}{c}\text { Clima } \\
\text { ego }\end{array}$ & $\begin{array}{c}\text { Cima } \\
\text { tarea }\end{array}$ \\
\hline Uso recompensas & $-.27^{* *}$ & -.11 & $-.24^{* *}$ & $.44^{* * *}$ & $-.32^{* * *}$ \\
Atención condición negativa & -.15 & .08 & $-.23^{*}$ & $.51^{* * *}$ & $-.27^{* *}$ \\
Intimidación & $-.25^{* *}$ & .04 & $-.30^{* * *}$ & $.57^{* * *}$ & $-.32^{* * *}$ \\
Control personal & $-.23^{*}$ & .04 & $-.25^{* *}$ & $.54^{* * *}$ & $-.35^{* * *}$ \\
Interés opinión deportista & .06 & $.32^{* * *}$ & .11 & .05 & .11 \\
Valoración comportamiento autónomo & $.21^{*}$ & $.30^{* * *}$ & $.27^{* *}$ & -.05 & .14 \\
\hline
\end{tabular}

$* p<.05 ; * * p<.01, * * * p<.001$

En la tabla 3 se muestran los modelos de regresión lineal (pasos sucesivos) que se han generado. Las variables predictoras han sido el estilo controlador del entrenador y el apoyo a la autonomía y las variables criterio las NPB y el clima motivacional. Las variables excluidas en los diversos casos no están presentes por falta de significación $(p>.05)$. Los datos cumplen los supuestos de linealidad en la relación entre variables predictoras y criterio, así como la homocedasticidad y distribución normal de los residuos, cuyo valor medio es 0 y la desviación típica prácticamente 1 (.99). Además, los valores de Durbin-Watson son satisfactorios, con rango entre 1.59 y 1.91 (Pardo y Ruiz, 2005).

El modelo de regresión para la satisfacción de la competencia explica el $6 \%$ de la varianza e incluye al predictor valoración del comportamiento autónomo del deportista $(F=7.77 ; p<.01)$. Para la satisfacción de la autonomía el modelo explica el $11 \%$ de la varianza e incluye al predictor interés por la opinión del deportista $(F=14.34 ; p<.001)$. Para la satisfacción de la relación con los demás el modelo explica el $16 \%$ de la varianza e incluye los predictores intimidación y valoración del comportamiento autónomo $(F=9.98 ; p<.001)$. Para el clima de implicación en el ego el modelo explica el
$48 \%$ de la varianza e incluye los predictores intimidación y atención de la condición negativa $(F=$ 53.68; $p<.001)$. Para el clima de implicación en la tarea el modelo explica el $33 \%$ de la varianza e incluye los predictores intimidación y atención de la condición negativa $(F=24.94 ; p<.001)$.

\section{DISCUSIÓN}

El propósito de este estudio consistió en analizar las relaciones entre los estilos de interacción (el estilo controlador y el apoyo a la autonomía) de los entrenadores con la percepción de clima motivacional y la satisfacción de las necesidades psicológicas básicas (competencia, autonomía y relación) en jóvenes futbolistas. Los datos han mostrado relaciones positivas entre el estilo de apoyo a la autonomía y la satisfacción de las necesidades psicológicas básicas. El estilo controlador se ha relacionado negativamente con la satisfacción de las necesidades psicológicas básicas y la percepción de clima de implicación en la tarea, así como relaciones positivas con el clima de implicación en el ego. En términos generales estos datos son congruentes con estudios que lo habían evaluado previamente (e.g., Cano, Montero, Cervelló, y Moreno-Murcia, 2018; Cantú-Berrueto, Castillo, López-Walle, Tristán, y Balaguer, 2016; Morillo, Reigal, y HernándezMendo, 2018). 


\section{Clima motivacional y necesidades psicológicas básicas en futbolistas jóvenes}

Tabla 3

Análisis de regresión lineal.

\begin{tabular}{|c|c|c|c|c|c|c|c|c|c|}
\hline $\begin{array}{l}\text { Variables } \\
\text { criterio } \\
\end{array}$ & $\mathrm{M}$ & $\mathrm{R}$ & $\mathrm{R}^{2}$ & $\begin{array}{l}\mathrm{D}- \\
\mathrm{W}\end{array}$ & $\begin{array}{l}\text { Variables } \\
\text { Predictoras } \\
\end{array}$ & Beta & $\mathrm{t}$ & $\mathrm{T}$ & FIV \\
\hline \multirow{2}{*}{$\begin{array}{l}\text { Necesidad } \\
\text { competencia }\end{array}$} & \multirow[t]{2}{*}{1} & \multirow[t]{2}{*}{.25} & \multirow[t]{2}{*}{.06} & \multirow[t]{2}{*}{1.59} & (Constante) & \multicolumn{3}{|c|}{$24.76 * * *$} & \\
\hline & & & & & Valoración & -.25 & $-2.79 * *$ & 1.00 & 1.00 \\
\hline \multirow{2}{*}{$\begin{array}{l}\text { Necesidad } \\
\text { autonomía }\end{array}$} & \multirow[t]{2}{*}{1} & \multirow[t]{2}{*}{.34} & \multirow[t]{2}{*}{.11} & \multirow[t]{2}{*}{1.73} & (Constante) & \multicolumn{3}{|c|}{$13.50 * * *$} & \\
\hline & & & & & Interés & .34 & $3.76 * * *$ & 1.00 & 1.00 \\
\hline \multirow{5}{*}{$\begin{array}{l}\text { Necesidad } \\
\text { relación con } \\
\text { los demás }\end{array}$} & \multirow[t]{2}{*}{1} & \multirow{2}{*}{.36} & \multirow[t]{2}{*}{.12} & \multirow[t]{2}{*}{1.61} & (Constante) & \multirow{2}{*}{\multicolumn{2}{|c|}{$\begin{array}{l}29.11^{* * *} \\
-3.81 * * *\end{array}$}} & & \\
\hline & & & & & Intimidación & & & 1.00 & 1.00 \\
\hline & \multirow[t]{3}{*}{2} & \multirow[t]{3}{*}{.42} & \multirow[t]{3}{*}{.16} & \multirow[t]{3}{*}{1.61} & (Constante) & & $10.59 * * *$ & & \\
\hline & & & & & Intimidación & -.32 & $-3.32 * * *$ & .96 & 1.05 \\
\hline & & & & & Valoración & .21 & $2.21 *$ & .96 & 1.05 \\
\hline \multirow[t]{5}{*}{ Clima ego } & \multirow[t]{2}{*}{1} & \multirow[t]{2}{*}{.66} & \multirow[t]{2}{*}{.43} & \multirow[t]{2}{*}{1.92} & (Constante) & \multirow{2}{*}{\multicolumn{2}{|c|}{$\begin{array}{l}15.62 * * * \\
9.40 * * *\end{array}$}} & & \\
\hline & & & & & Intimidación & & & 1.00 & 1.00 \\
\hline & \multirow[t]{3}{*}{2} & \multirow[t]{3}{*}{.70} & \multirow[t]{3}{*}{.48} & \multirow[t]{3}{*}{1.92} & (Constante) & & $11.65 * * *$ & & \\
\hline & & & & & Intimidación & .45 & $4.85 * * *$ & .53 & 1.89 \\
\hline & & & & & $\begin{array}{l}\text { Atención condición } \\
\text { negativa }\end{array}$ & .31 & $3.34 * * *$ & .53 & 1.79 \\
\hline \multirow[t]{5}{*}{ Clima tarea } & \multirow[t]{2}{*}{1} & .55 & .30 & 1.91 & (Constante) & & $56.86^{* * *}$ & & \\
\hline & & & & & Intimidación & -.55 & $-6.59 * * *$ & 1.00 & 1.00 \\
\hline & 2 & .58 & .33 & 1.91 & (Constante) & & $49.30 * * *$ & & \\
\hline & & & & & Intimidación & -.39 & $-3.50 * * *$ & .55 & 1.83 \\
\hline & & & & & Atención $\mathrm{CN}$ & -.24 & $-2.19 *$ & .55 & 1.83 \\
\hline
\end{tabular}

El estilo controlador se relacionó negativamente con la satisfacción de la competencia y la relación con los demás, aunque no con la satisfacción de la autonomía. Los entrenadores que poseen un estilo interactivo controlador son menos flexibles y muestran menor empatía con los deportistas (Mars et al., 2017). Estoy podría provocar una menor supervisión del progreso de cada jugador, no siendo sensible a las necesidades de cada uno de ellos. Además, en contextos de mucha presión y poco espacio para comunicar cómo se sienten o qué necesitan, los deportistas tienen menos posibilidad de encontrar vías para el desarrollo personal y social, lo cual podría incidir en la percepción de la propia competencia y de la posibilidad de generar interacciones satisfactorias con otras personas. Así, tal y como ocurre en este trabajo, existe una mayor probabilidad de dificultar la satisfacción de las necesidades psicológicas básicas (Castillo et al., 2012).

Por el contrario, los estilos que favorecen la autonomía generan contextos adecuados para el desarrollo deportivo y personal, facilitando la comunicación entre las personas y aceptando la opinión del deportista (Bartholomew et al., 2010). En 


\section{Moreno-Luque, M., Reigal, R. E., Morillo-Baro, J. P., Morales-Sánchez, V., Hernández-Mendo, A.}

estudios previos se ha observado que la promoción de la autonomía en el jugador permite incrementar su bienestar, favorece su funcionamiento y permite, específicamente, satisfacer sus necesidades psicológicas básicas (e.g., Álvarez, Estevan, Falcó, y Castillo, 2013; Morillo et al., 2018; Schüler et al., 2014). Este fenómeno se ha reproducido en el presente trabajo, siendo la valoración del comportamiento autónomo predictor de las puntuaciones en satisfacción de la competencia y el interés por la opinión del deportista predictor de la satisfacción de la autonomía. Sin embargo, tal y como sí ha sucedido en estudios anteriores (SáenzLópez, Mateos, Almagro, y Conde, 2017), el apoyo a la autonomía no se ha relacionado significativamente con la percepción del clima motivacional de implicación en el ego o en la tarea, siendo una limitación del presente trabajo.

El estilo controlador sí se ha relacionado con la percepción del clima motivacional de implicación al ego o a la tarea, coincidiendo con estudios anteriores (Cano et al., 2018). Los entrenadores controladores suelen ser coercitivos y autoritarios (Bartholomew et al., 2010), no suelen centrarse en las necesidades del deportista ni promocionan su motivación intrínseca, por lo que podría disminuir la percepción motivacional centrada en la tarea. Se centran más en desarrollar sus ideas $y$ atienden menos las necesidades de los deportistas y suelen orientar más la práctica deportiva en el rendimiento deportivo, sin tener en cuenta la satisfacción o bienestar de sus jugadores (Castillo-Jiménez, López-Walle, Tomás, y Balaguer, 2017). De ahí que, tal y como se puede observar en este trabajo y siendo coherente con las ideas previas expuestas sobre este tema, el estilo controlador del entrenador se haya relacionado positivamente con la percepción de clima motivacional de implicación en el ego y dimensiones como intimidación y atención condición negativa hayan sido excelentes predictores de sus puntuaciones.

Entre las limitaciones de este trabajo se encuentra el tamaño de la muestra, la cual no permite efectuar análisis más precisos por edad o género. Ampliar su tamaño en futuros estudios permitirá seguir profundizando sobre cómo afecta este fenómeno en diferentes categorías deportivas y si ocurre igual entre chicos y chicas. Además, sería interesante enfocar las próximas investigaciones desde los modelos teóricos que conceptualizan los climas motivaciones como empowering y disempowering, la cual permite observarlo desde una perspectiva más completa. A pesar de ellas, este trabajo permite seguir obteniendo datos sobre un ámbito de conocimiento esencial para el deporte actual, sugiriendo a los entrenadores reflexionar sobre su práctica profesional y valorar el contexto de práctica que generan.

\section{REFERENCIAS}

1. Adame-Garza, O., Tristán-Rodríguez, J., Tomás, I., Hernández-Mendo, A., y López-Walle, J. (2017). Presentación de las tareas, satisfacción de las necesidades psicológicas básicas y bienestar en Atletas universitarios. Journal of Behavior, Health \& Social Issues, 9(1), 13-20. https://doi.org/10.1016/j.jbhsi.2017.06.003

2. Álvarez, M., Castillo, I., Duda, J., y Balaguer, I. (2009). Clima motivacional, metas de logro y motivación autodeterminada en futbolistas cadetes. Revista de Psicología General y Aplicada, 62(1-2), 35-44.

3. Álvarez, O., Estevan, I., Falcó, C., y Castillo, I. (2013). Efectos del apoyo a la autonomía del entrenador en taekwondistas españoles de élite. Revista Iberoamericana de Psicología del Ejercicio y el Deporte, 8(1), 59-70.

4. Atkins, M.R., Johnson, D.M., Force, E.C., y Petrie, T.A. (2015). Peers, parents, and coaches, oh my! The relation of the motivational climate to boys' intention to continue in sport. Psychology of Sport \& Exercise, 16(3), 170-180.

5. Balaguer, I., González, L., Fabra, P., Castillo, I., Mercé, J., y Duda, J. L. (2012). Coaches' interpersonal style, basic psychological needs, and the well- and ill-being of young soccer players: A longitudinal analysis. Journal of Sports Sciences, 30(15), 1619-1629. http://dx.doi.org/10.1080/02640414.2012.731517

6. Balaguer, I., Guivernau, M., Duda, J. L., y Crespo, M. (1997). Análisis de la validez de constructo y de la validez predictiva del cuestionario de clima motivacional percibido en el deporte (PCMSQ-2) con tenistas españoles de 


\section{Clima motivacional y necesidades psicológicas básicas en futbolistas jóvenes}

competición. Revista de Psicología del Deporte, 6(1), 41-58.

7. Barbosa-Luna, A. E., Tristán, J. L., Tomás, I., González, A., y López-Walle, J. M. (2017). Climas motivacionales, motivación autodeterminada, afectos y burnout en deportistas: enfoque multinivel. Acción Psicológica, 14(1), 105-118. https://doi.org/10.5944/ap.14.1.19266

8. Bartholomew, K. J., Ntoumanis, N., y Thøgersen-Ntoumani, C. (2010). The controlling interpersonal style in a coaching context: Development and initial validation of a psychometric scale. Journal of Sport \& Exercise Psychology, $\quad 32, \quad$ 193-216. https://doi.org/10.1123/jsep.32.2.193

9. Bullock, T., y Giesbrecht, B. (2014). Acute exercise and aerobic fitness influence selective attention during visual search. Frontiers in Psychology, 5, 1290. https://doi.org/10.3389/fpsyg.2014.01290

10. Cano, F., Montero, C., Cervelló, E., y MorenoMurcia, J. A. (2018). Influencia del estilo interpersonal del entrenador sobre la motivación en deportistas de salvamento y socorrismo. Cuadernos de Psicología del Deporte, 18(1), 173-182.

11. Cantú-Berrueto, A., Castillo, I., López-Walle, J., Tristán, J., y Balaguer, I. (2016). Estilo interpersonal del entrenador, necesidades psicológicas básicas y motivación: un estudio en futbolistas universitarios mexicanos. Revista Iberoamericana de Psicología del Ejercicio y el Deporte, 11(2), 263-270.

12. Castillo, I., Duda, J. L., Álvarez, M. S., Mercé, J., y Balaguer, I. (2011). Clima motivacional, metas de logro de aproximación y evitación y bienestar en futbolistas cadetes. Revista de Psicología del Deporte, 20(1), 149-164.

13. Castillo, I., González, L., Fabra, P., Mercé, J., y Balaguer, I. (2012). Estilo interpersonal controlador del entrenador, frustración de las necesidades psicológicas básicas, y burnout en futbolistas infantiles. Cuadernos de Psicología del Deporte, 12(1), 143-146. https://doi.org/10.4321/S157884232012000100014
14. Castillo, I., Tomás, I., Ntoumanis, N., Bartholomew, K., Duda, J., y Balaguer, I. (2014). Psychometric properties of the Spanish version of the Controlling Coach Behaviors Scale in the sport context. Psicothema: Revista de Psicologia, 26(3), 409-414. https://doi.org/10.1037/t61581-000

15. Castillo-Jiménez, N., López-Walle, J. M., Tomás, I., y Balaguer, I. (2017). Relación del clima empowering con la motivación autodeterminada a través de la satisfacción de las necesidades psicológicas básicas. Revista de Psicología del Deporte, 26(3), 33-39.

16. Conde, C., Sáenz-López, P., Carmona, J., González-Cutre, D., Martínez, C., y Moreno, J. A. (2010). Validación del Cuestionario de Percepción de Soporte de la Autonomía en el Proceso de Entrenamiento (ASCQ) en jóvenes deportistas españoles. Estudios de Psicología, 31(2), 145-157. https://doi.org/10.1174/021093910804952250

17. Conroy, D.E., y Coatsworth, J.D. (2006). Coach training as a strategy for promoting youth social development. The Sport Psychologist, 20,128144. https://doi.org/10.1123/tsp.20.2.128

18. Deci, E. L. y Ryan, R. M. (1985). Intrinsic motivation and self-determination in human behavior. Nueva York: Plenum Press. https://doi.org/10.1007/978-1-4899-2271-7

19. Deci, E. L. y Ryan, R. M. (1987). The support of autonomy and the control of behavior. Journal of Personality and Social Psychology, 53, 10241037. https://doi.org/10.1037/00223514.53.6.1024

20. Deci, E. L. y Ryan, R. M. (2000). The "what" and "why" of goal pursuits: Human needs and the self-determination of behaviour. Psychological Inquiry, 11, 227-268. https://doi.org/10.1207/S15327965PLI1 104_01

21. Duda, J. L. (2001). Achievement goal research in sport: Pushing the boundaries and clarifying some misunderstandings. En G. C. Roberts (Ed.), Advances in motivation in sport and exercise (pp. 129-182). Champaign, IL: Human Kinetics.

22. Duda, J. L. y Balaguer, I. (2007). Coach-created motivational climate. En S. Jowett y D. Lavallee 


\section{Moreno-Luque, M., Reigal, R. E., Morillo-Baro, J. P., Morales-Sánchez, V., Hernández-Mendo, A.}

(Eds.), Social psychology in sport (pp. 117-130). Champaign, IL: Human Kinetics.

23. Duda, J. L. y Nicholls, J. G. (1992). Dimensions of achievement-motivation in schoolwork and sport. Journal of Educational Psychology, 84(3), 290-299. $\quad$ https://doi.org/10.1037/00220663.84.3.290

24. Fabra, P., Balaguer, I., Castillo, I., Mercé, J., y Duda, J. L. (2013). La eficacia de rol como mediadora entre el clima motivacional y el rendimiento en jóvenes futbolistas. Revista de Psicología Social, 28(1), 47-58. https://doi.org/10.1174/021347413804756023

25. García-Calvo, T., Sánchez-Miguel, P. A., LeoMarcos, F. M., Sánchez-Oliva, D., y Amado, D. (2012). Análisis del grado de diversión e intención de persistencia en jóvenes deportistas desde la perspectiva de la teoría de la autodeterminación. Revista de Psicología del Deporte, 21(1), 7-13. https://doi.org/10.5232/ricyde2011.02502

26. González, L., Tomás, I., Castillo, I., Duda, J. L., y Balaguer, I. (2017). A test of basic psychological needs theory in young soccer players: time-lagged design at the individual and team levels. Scandinavian Journal of Medicine \& Science in Sports, 27(11), 1511-1522. https://doi.org/10.1111/sms.12778

27. Jaakkola, T., Ntoumanis, N., y Liukkonen, J. (2016). Motivational climate, goal orientation, perceived sport ability, and enjoyment within F innish junior ice hockey players. Scandinavian Journal of Medicine \& Science in Sports, 26(1), 109-115. https://doi.org/10.1111/sms. 12410

28. Mars, L., Castillo, I., López-Walle, J. y Balaguer, I. (2017). Estilo controlador del entrenador, frustración de las necesidades y malestar en futbolistas. Revista de Psicología del Deporte, 26(2), 119-124.

29. Moreno-Murcia, J. A., Marzo, J. C., MartínezGalindo, C., y Conte Marín, L. (2011). Validación de la Escala de" Satisfacción de las Necesidades Psicológicas Básicas. RICYDE. Revista Internacional de Ciencias del Deporte, 7(26), 355-369. https://doi.org/10.5232/ricyde2011.02602
30. Morillo, J. P., Reigal, R. E., y Hernández-Mendo, A. (2018). Orientación motivacional, apoyo a la autonomía y necesidades psicológicas en balonmano playa. Revista Internacional de Medicina y Ciencias de la Actividad Física y el Deporte, 18(69), 103-117.

31. Newton, M., Duda, J. L., y Yin, Z. (2000). Examination of the psychometric properties of the Perceived Motivational Climate in Sport Questionnaire-2 in a sample of female athletes. Journal of Sports Sciences, 18(4), 275-290. https://doi.org/10.1080/026404100365018

32. Nicholls, J. G. (1989). The competitive ethos and democratic education. Cambridge, MASS: Harvard University Press.

33. Olympiou, A., Jowett, S., y Duda, J. L. (2008). The psychological interface between the coachcreated motivational climate and the coachathlete relationship in team sports. The Sport Psychologist, 22(4), 423-438. https://doi.org/10.1123/tsp.22.4.423

34. Orkibi, H., y Ronen, T. (2017). Basic psychological needs satisfaction mediates the association between self-control skills and subjective well-being. Frontiers in Psychology, 8, 936. https://doi.org/10.3389/fpsyg.2017.00936

35. Pardo, A. y Ruiz, M. A. (2005). Análisis de datos con SPSS 13 Base. Madrid: McGraw Hill.

36. Rhodes, R. E., Janssen, I., Bredin, S. S., Warburton, D. E., y Bauman, A. (2017). Physical activity: Health impact, prevalence, correlates and interventions. Psychology \& Health, 32(8), 942-975.

https://doi.org/10.1080/08870446.2017.1325486

37. Sáenz-López, P., Mateos, J. L., Almagro, B. J., y Conde, C. (2017). Apoyo a la autonomía, creencias implícitas de habilidad y metas de logro en jugadoras de baloncesto en formación. Cuadernos de Psicología del Deporte, 17(3), 199-206.

38. Schüler, J., Wegner, M. y Knechtle, B. (2014). Implicit motives and basic need satisfaction in extreme endurance sports. Journal of Sport and Exercise Psychology, 36(3), 293-302. https://doi.org/10.1123/jsep.2013-0191 


\section{Clima motivacional y necesidades psicológicas básicas en futbolistas jóvenes}

39. Smith, N., Quested, E., Appleton, P. R., y Duda, J. L. (2017). Observing the coach-created motivational environment across training and competition in youth sport. Journal of Sports Sciences, 35(2), 149-158. https://doi.org/10.1080/02640414.2016.1159714

40. Sylvester, B. D., Curran, T., Standage, M., Sabiston, C. M., y Beauchamp, M. R. (2018). Predicting exercise motivation and exercise behavior: A moderated mediation model testing the interaction between perceived exercise variety and basic psychological needs satisfaction. Psychology of Sport and Exercise, 36, 50-56. https://doi.org/10.1016/j.psychsport.2018.01.004

41. Torregrosa, M., Viladrich, C., Ramis, Y., Azócar, F., Latinjak, A., y Cruz, J. (2010). Efectos en la percepción del clima motivacional generado por los entrenadores y compañeros sobre la diversión y el compromiso. Diferencias en función de género. Revista de Psicología del Deporte, 20(1), 243-255.

42. Warburton, D. E. R., y Bredin, S. S. D. (2017). Health benefits of physical activity: a systematic review of current systematic reviews. Current Opinion in Cardiology, 32(5), 541-556. https://doi.org/10.1097/HCO.0000000000000437

43. Wilson, P. M., Rogers, W. T., Rodgers, W. M., y Wild, T. C. (2006). The psychological need satisfaction in exercise scale. Journal of Sport and Exercise Psychology, 28(3), 231-251. https://doi.org/10.1123/jsep.28.3.231

44. World Medical Association. Declaration of Helsinki (2013). Disponible en http://www.wma.net/en/20activities/10ethics/10h elsinki/, [Consulta: 23 de marzo de 2015]. 